\title{
De la comadrona a la obstetriz. Nacimiento y apogeo de la profesión de partera titulada en el Perú del siglo XIX
}

\author{
Lissell Quiroz (*) \\ ${ }^{*}$ ) Université de Rouen, Francia. \\ lissell.quiroz-perez@univ-rouen.fr
}

Dynamis

[0211-9536] 2012; $32(2):$ 415-437

Fecha de recepción: 22 de noviembre de 2011

Fecha de aceptación: 29 de marzo de 2012

SUMARIO: 1.-Introducción. 2.-La herencia ilustrada: denigración de la matrona y desarrollo de la obstetricia. 3.-Benita Paulina Cadeau, la «Madame Lachapelle» peruana. 4.- Una formación teórica y práctica innovadora en el mundo hispánico. 5.-Desarrollo de la profesión de obstetriz bajo la tutela de los médicos. 6.-Conclusión.

RESUMEN: En el Perú como en el resto del mundo hispánico, el siglo XIX fue un momento de profunda transformación del ejercicio de la medicina y particularmente de la obstetricia. Unas de las actrices principales de esta evolución fueron las parteras tituladas cuya profesión nació y se asentó entonces. Hasta ahí, las matronas tradicionales eran las que se encargaban de acompañar a las parturientas, en un universo prácticamente totalmente femenino. A finales del siglo XVIII, los ilustrados peruanos comenzaron a interesarse en la maternidad y el periodo perinatal. Fustigaron principalmente la práctica de las parteras tradicionales y expusieron ante la opinión pública, la necesidad de darles una formación teórica que estuviera bajo la supervisión del cuerpo médico. El discurso ilustrado fue retomado después de la independencia por las autoridades públicas del Estado naciente. El proyecto se concretó gracias a la llegada al Perú de una partera francesa de excepción, Benita Paulina Fessel, mujer emprendedora deseosa de fundar una maternidad bajo el modelo de la de Port-Royal (París). La confluencia de estos factores permitió el nacimiento en 1826 de la primera Maternidad del mundo hispánico con un funcionamiento particular que asociaba un hospital y una escuela de partos, dirigida por Madame Fessel, ella misma ex alumna de la Maternidad de París. La maternidad limeña formó varias generaciones de parteras, que recibieron una excelente formación tanto teórica como práctica y que se impusieron como profesionales competentes. Durante la segunda mitad del siglo XIX las obstetrices, como se les llama desde esa época en Perú, acompañaron cada vez a más mujeres en el trance del parto y resistieron a la presión de los médicos por apropiarse de ese momento esencial en la vida de las mujeres.

PALABRAS CLAVE: Perú, parteras, obstetricia, profesión médica, siglo XIX.

KEY WORDS: Peru, midwives, obstetrics, medical profession, 19th century. 


\section{Introducción}

Durante mucho tiempo, nuestras historias de la medicina y, más concretamente, de la maternidad y la obstetricia estuvieron centradas en las hazañas de los grandes médicos conformando una perspectiva teleológica de la ciencia. Afortunadamente, desde las últimas décadas del siglo XX una serie de investigaciones, incluyendo algunas aportaciones españolas, han tratado de cambiar este ángulo de visión, fomentando el estudio de la historia de la maternidad y del papel de las mujeres en la historia de la obstetricia. Uno de los temas más destacados ha sido el de la historia de las matronas ${ }^{1}$. El interés por este tema ha pasado a Hispanoamérica, como lo muestra una amplia bibliografía hoy disponible ${ }^{2}$. Y con justa razón pues estas actrices de la historia, en un principio menospreciadas y luego olvidadas, ocuparon siempre un lugar estratégico no solo en el periodo perinatal sino también en

1. Una serie de trabajos europeos han sido publicados en la década de los 80: Pancino, Claudia. Il bambino e l'acqua sporca. Storia dell'assitenza al parto delle mammane alle ostetriche (secoli XVI-XX). Milán: Franco Angeli; 1984; Donnison, Jean. Midwifes and medical men. A history of the struggle for the control of childbirth. Londres: Historical Publications; 1988; Gélis, Jacques. La sage-femme ou le médecin: une nouvelle conception de la vie. París: Fayard; 1988; Marland, Hilary, ed. The art of midwifery. Early modern midwives in Europe. London: Routledge, 1993; Marland, Hilary; Rafferty, Anne Marie, eds. Midwives, society and childbirth. Debates and controversies in the modern period, London: Routledge; 1997. El caso español ha sido estudiado en: Ortiz Gómez, Teresa. From hegemony to subordination: midwives in early modern Spain. In: Marland, n. 1, 1993, p. 95-114; Luisa Rosado o el orgullo de ser matrona en la España ilustrada. Dynamis. 1992; 12: 323-346; Las matronas y la transmisión de saberes científicos sobre el parto en la España del siglo XIX. Arenal. 1999; 6 (1): 55-79; De matrona a matrona: Francisca Iracheta y la divulgación de la ciencia obstétrica en España en 1870. Arenal. 1999; 6 (1): 183-195; Ortiz Gómez, Teresa; Martínez Padilla, Clara. How to be a midwife in late ninetheenth-century Spain. In: Marland, n. 1, 1997, p. 61-80; Cabré i Pairet, Montserrat; Ortiz Gómez, Teresa, eds. Sanadoras, matronas y médicas en Europa, siglos XII-XX. Barcelona: Icaria, 2001.

2. Carrillo, Ana María. Nacimiento y muerte de una profesión. Las parteras tituladas en México. Dynamis. 1999; 19: 167-190; Agostoni, Claudia. Médicos y parteras en la ciudad de México durante el Porfiriato. In: Cano, Gabriela; Valenzuela, Georgette José. Cuatro estudios de género en el México urbano del siglo XIX. México: UNAM-PUEG; 2001, p. 71-95; Díaz Robles, Laura Catalina; Oropeza Sandoval, Luciano. Las parteras de Guadalajara (México) en el siglo XIX: el despojo de su arte. Dynamis. 2007; 27: 237-261; Alanís Rufino, Mercedes. Una cuestión de parteras y médicos. Mujeres en el Hospital de la Maternidad e Infancia en la Ciudad de México, 1861-1905. Boletín Mexicano de Historia y de Filosofía de la Medicina. 2009: 12 (2): 63-68; Restrepo, Libia J. Médicos y comadronas o el arte de los partos. La ginecología y la obstetricia en Antioquia, 1870-1930. Medellín: La Carreta Ed.; 2006; Lattus Olmos, José; Sanhueza Benavente, María Carolina. La matrona y la obstetricia en Chile, una reseña histórica. Revista Chilena de Obstetricia y Ginecología. 2007; 2 (3): 271-276. 
muchos momentos de la vida de las mujeres. Desde un punto de vista social, su poder fue tradicionalmente importante pues dominaban un ámbito al que los hombres no tuvieron realmente acceso hasta finales del siglo XVIII.

La entrada en el siglo XIX marcó una ruptura en la historia de la obstetricia. Primeramente, los avances médicos permitieron la apertura de un campo hasta allí bastante desdeñado por los facultativos. El discurso ilustrado, con sus ataques cada vez más contundentes a las parteras tradicionales, preparó un terreno favorable al nacimiento de una nueva profesión, la de las matronas tituladas. Estas transformaciones se produjeron en un clima de competencia - a veces cordial y otras menos- entre las parteras tradicionales, las tituladas, los médicos y las autoridades, quienes se disputaron el dominio del campo de la maternidad. El caso peruano ha sido hasta aquí poco estudiado ${ }^{3}$. Todas las obras referentes a la historia de la medicina peruana aluden a la labor de la primera partera titulada del Perú, Benita Paulina Cadeau-Fessel, fundadora de la Maternidad de Lima ${ }^{4}$. Pero si bien se ha estudiado la biografía y la trayectoria de esta partera, carecemos de análisis detallados de sus escritos, excepcionales pues pocas matronas del mundo hispánico escribieron tanto como ella. Tampoco existe ningún estudio de las demás parteras, aquellas que siguieron las enseñanzas de Madame Fessel y las que le sucedieron. El presente trabajo trata de resaltar la participación histórica de más de 260 parteras peruanas que ejercieron su profesión entre 1826 y 1902. Se trata también de estudiar la originalidad del modelo peruano en relación a otros sistemas latinoamericanos o europeos, pues Lima fue la primera ciudad hispana en organizar un sistema de formación de parteras diplomadas asociado a una Maternidad, tomando como modelo la de París. Apenas asentada la independencia en 1826, el gobierno peruano optó por establecer y controlar la profesión de

3. El único investigador que ha trabajado esta cuestión es Miguel Rabí Chara. El Hospital de la Maternidad de Lima y la Escuela de Obstetrices del Perú (1826-1836). Labor y obra de Benita Paulina Cadeau de Fessel. Lima: Grahuer; 2004. En este volumen el autor, que no es historiador, presenta una historia de la maternidad desde la época de la colonia hasta la partida de Madame Fessel del Perú en 1836. La mayor parte del libro corresponde a la recopilación de los textos escritos por la matrona francesa; por consiguiente, se trata de una historia más factual que analítica, interesante porque proporciona datos que el autor encontró en los archivos del Ministerio de Salud, donde trabajó durante largos años, pero que merece ser completada.

4. El apellido de soltera de esta matrona francesa es Cadeau. Al casarse, se le agregó el apellido de su esposo, Fessel, con el que fue más conocida en el Perú, donde adquirió el apelativo de «Madama Fessel». 
obstetriz - como se designa a la matrona en Perú - siguiendo los preceptos establecidos desde finales del siglo XVIII. La formación de cuatro años asociada a la práctica en la Maternidad hicieron de las matronas peruanas unas profesionales altamente calificadas que acompañaron a cientos de mujeres peruanas en el trance crucial del parto, desempeñando un papel determinante en la historia de la obstetricia peruana.

Desafortunadamente, no disponemos de todas las fuentes necesarias para este estudio. Hasta hace muy poco, la historia de las mujeres y más la historia de la maternidad era vista con cierto desprecio y desinterés, contrariamente a la de los médicos. Así, los archivos de la Maternidad de Lima, conservados en las bóvedas del hospital hasta 1970, fueron literalmente desechados y hasta quemados pues no parecían dignos de interés. Hay entonces que orientarse hacia otro tipo de fondos como los del Archivo General de la Nación, los de la Facultad de Medicina, del Instituto RivaAgüero o de la Biblioteca Nacional. El estudio de estos fondos nos permite interrogarnos sobre el papel de las parteras diplomadas, las relaciones de género y la historia de la salud a través del prisma de la maternidad. Trataremos de considerar todos estos aspectos en este artículo.

\section{La herencia ilustrada: denigración de la matrona y desarrollo de la obstetricia}

Las últimas décadas del siglo XVIII estuvieron marcadas por un importante desarrollo de la ciencia y principalmente de la medicina, disciplina cada vez más valorada socialmente. En un artículo sobre la maternidad y la medicina en el Perú del siglo XVIII, Claudia Rosas muestra cómo los intelectuales de la Ilustración peruana se interesaron de manera muy activa en la maternidad con un objetivo netamente pedagógico ${ }^{5}$. Obsesionados con la ciencia y la razón, los ilustrados peruanos plasmaron una mirada negativa y despreciativa del periodo perinatal. La parturienta aparecía entonces como un ser dominado por fuerzas primitivas e irracionales. En la representación médica del Antiguo Régimen la mujer, principalmente durante el embarazo, podía ser vista como un ser dominado por su útero

5. Rosas, Claudia. Madre solo hay una. Ilustración, maternidad y medicina en el Perú del siglo XVIII. Anuario de estudios americanos. Escuela de estudios hispano-americanos. 2004: 61 (1): 103-138. 
como lo sintetiza el axioma del doctor Van Helmont: propter solum uterum mulier est id quod est. En ese mundo de irracionalidad y de histeria, el médico se presentó como el más indicado para intervenir y sustituir al cura exorcista. Podemos ver paralelamente un interés cada vez mayor por la obstetricia, disciplina médica hasta entonces menospreciada. Así por ejemplo el Mercurio Peruano, editado por un grupo de jóvenes de la Sociedad de Amantes del País, consagró varios artículos a este tema. Muchos de ellos dictaban reglas que debían observarse durante el embarazo al mismo tiempo que exhortaban a las mujeres a amamantar a sus hijos. Pero la crítica más importante de la época se dirigió contra las matronas, consideradas sucias, incultas y supersticiosas. Se les imputaba su ignorancia así como su baja extracción, como lo resume uno de los más célebres colaboradores del Mercurio Peruano, el médico y científico Hipólito Unanue (1755-1833) en un discurso pronunciado en la Universidad de San Marcos:

«A imitación de los hombres sin instrucción ni conciencia, que encontraron su subsistencia en la práctica de la medicina, unas mujeres incapaces y por lo regular de esfera humilde, se apoderaron de la delicada parte de la cirugía, que cuida del exordio de la humanidad; del arte de partear, cuyo ejercicio pide virtud, calidad y ciencia. El ningún freno y abandono formó una plaga no menos sangrienta que la primera» ${ }^{6}$.

La diatriba en contra de la matrona tradicional tenía por contrapeso la alabanza del médico. A partir de ahí, tanto la figura del médico como su presencia en el momento del parto se fueron reforzando. Surgió entonces la idea de controlar y de vigilar de cerca a las matronas utilizando para ello a los cirujanos, de los cuales éstas dependían tradicionalmente. Los ilustrados eran unánimes sobre este punto y hasta religiosos como el padre Francisco González de Laguna insistieron en la necesidad de comprometer a los médicos en esta tarea:

«La elección debía hacerse de las más juiciosas, prudentes, devotas y honradas que se hallen, que sean casadas o viudas, y no las que hubieran vivido en la prostitución, para evitar las maldades en que están habituadas

6. Discurso pronunciado en la Real Universidad de Lima el día 21 de noviembre de 1792, en la inauguración del Anfiteatro Anatómico en el Hospital San Andrés de Lima. In: Obras científicas y literarias de D. Hipólito Unanue [Colección Clásicos de la Medicina Peruana, vol. 2], Barcelona: Consultoría y Equipamientos Médicos S. A.; 1914, p. 21. 
estas personas y será fácil que inspiren a sus clientes; que sean sanas y robustas, que sean atentas, aseadas, no melancólicas y sobre todo exentas del mal venéreo. Debían instruirse en el arte, y para ello prescribirles una cartilla o tratado breve, y señalarles un cirujano hábil que cuide de su instrucción» ${ }^{7}$.

Hasta mediados del siglo XVIII, a pesar de que las matronas dependían de los cirujanos y por ende del Protomedicato, era al tribunal de la Inquisición al que le correspondía juzgar a aquellas mujeres acusadas de brujería y de prácticas mágicas ${ }^{8}$. Pero en 1750 la Corona ordenó que las matronas fueran examinadas y controladas por el Real Protomedicato de Madrid $^{9}$. Se encargó entonces la redacción de cartillas destinadas a la formación de las parteras en las que se les ordenaba recurrir a los médicos en caso de partos complicados. ¿Qué acogida tuvieron estas disposiciones en América? En México, las parteras se resistieron a estas disposiciones ${ }^{10}$. En el Perú, las ordenanzas se registraron y se archivaron pero en la práctica pocas cosas cambiaron. Varios factores explican esta inercia. Por un lado, las matronas no sintieron la necesidad de pasar un examen ante el cuerpo médico pues seguían siendo las especialistas del nacimiento, aquellas a las que se llamaba primero que nadie. Aunque se sabían criticadas por el discurso ilustrado, eran conscientes de su experiencia y de la desconfianza de las mujeres embarazadas hacia los médicos. Poseían un saber práctico que en muchos casos, sobre todo en los partos naturales, bastaba para acompañar a las parturientas. Cuando el alumbramiento se complicaba, recurrían a prácticas mágicas para conjurar lo que no podían controlar. Por otro lado, los médicos mostraban muy poco interés en la obstetricia que durante mucho tiempo fue una rama subalterna y poco valorada de la medicina. Además, todo separaba a las matronas, en su mayoría indígenas, negras o mestizas, generalmente incultas - es decir sin instrucción-de los médicos cuyo saber teórico estaba muy alejado de la sensibilidad femenina. El saber médico era por lo demás, en esa época, tan incompleto como el de las parteras tradicionales pues a pesar de los progresos de la obstetricia y de la literatura médica europea que llegaba a manos de los doctores,

7. González Laguna, P. Francisco. El zelo sacerdotal para con los niños no nacidos (...) va al fin un apéndice sobre la curación de los ahogados. Lima: Impr. de los Niños Expósitos; 1781, p. 99.

8. Lanning, John Tate. The Royal Protomedicato. The regulations of medical profession in the Spanish Empire. Durkham: Duke University Press; 1985.

9. Agostoni, n. 2, p. 78.

10. Carrillo, n. 2. 
éstos carecían de experiencia clínica. Tanto es así que hasta después de la Independencia ninguna matrona fue examinada por el Protomedicato.

En suma, a principios del siglo XIX, existía un terreno propicio para el surgimiento de parteras formadas en obstetricia y tituladas ${ }^{11}$. En un primer momento la prioridad del nuevo Estado se orientó hacia la consolidación de la independencia, efectiva y definitiva a principios de 1825. Ahora bien, por esa época, Hipólito Unanue fue nombrado presidente del Consejo de Gobierno. Casualmente ese mismo año, una partera francesa, Benita Paulina Cadeau-Fessel, se embarcó rumbo al Perú.

\section{Benita Paulina Cadeau, la «Madame Lachapelle» peruana}

Benita Paulina Cadeau nació en Lyon en 1792. Cursó sus estudios de obstetricia en el Hospicio de la Maternidad de París (Maternidad de Port-Royal) que había abierto sus puertas en el año 1802. Esto significa que había recibido una formación de gran calidad en una institución modelo en Europa que se caracterizaba por la asociación de la teoría y la práctica ${ }^{12}$. En esa época, los estudios de obstetricia duraban dos años, y ella estudió en la Maternidad de Port-Royal entre julio de 1816 y junio de $1818^{13}$. Era por consiguiente una partera de primera clase, habilitada para ejercer en toda Francia, frente a las parteras de segunda clase formadas en los hospicios departamentales ${ }^{14}$. Como señala la historiadora francesa Scarlett Beauvalet, la selección y la rigurosa formación de las alumnas hacían de éstas últimas un cuerpo de élite. Las parteras con título de Port-Royal eran conscientes de esa distinción como lo muestra el caso de Benita Paulina Cadeau. Por donde iba, presentaba su título de la Maternidad de París así como las

11. En el Perú actual, la obstetricia es una especialidad médica estudiada por dos tipos de profesionales: los tocólogos y las matronas. De modo tal que la palabra obstetra designa a la vez al tocólogo y a la matrona (también llamada obstetriz). Esta terminología deriva del siglo XIX, de la época del surgimiento de las parteras tituladas. En la Facultad de Medicina de Lima, existieron a partir de la década de 1850 cuatro secciones correspondientes a las diferentes profesiones médicas: medicina, farmacia, odontología y obstetricia. Ésta última correspondía a los estudios conocidos en España con el nombre de matronería. En este artículo hemos optado por conservar la terminología peruana.

12. Beauvalet-Boutouyrie, Scarlett. Naître à I'hôpital au XIXe siècle. Paris: Belin; 1999.

13. Fessel, Benita Paulina. Consejos a las mujeres encinta (sic). Guadalajara: Impr. de D. M. Rodríguez; 1825, p. II.

14. Beauvalet-Boutouyrie, n. 12, p. 112. 
condecoraciones obtenidas durante su pasantía: la medalla de obstetricia, el primer premio de observación sobre los diferentes caracteres de la vacuna y por último, el primer premio de botánica ${ }^{15}$. Se trataba de galardones establecidos por la Escuela de Partos para poner a prueba los conocimientos de las alumnas y favorecer al mismo tiempo la emulación entre ellas. Las estudiantes galardonadas obtenían de esta manera el reconocimiento de sus pares así como de sus superiores, como la matrona jefa o el médico jefe. Benita Paulina Cadeau estaba destinada a una buena carrera, sobre todo porque fue la alumna de una matrona muy activa y reconocida en el medio, Marie-Louise Lachapelle (1769-1821), quien desempeñó un papel muy importante en la organización y la reputación de la escuela parisina. La fama de esta matrona fue consecutiva a la calidad de su enseñanza, su competencia así como la abnegación y benevolencia que manifestaba para con sus pacientes ${ }^{16}$. Benita Paulina Cadeau se inspiró mucho en Madame Lachapelle, de la que siempre se reclamó discípula ${ }^{17}$.

Sin embargo, para finales de los años 1810 no era fácil ocupar una plaza de partera jefa pues había muchas candidatas y pocas vacantes. Benita Paulina Cadeau deseó probablemente ocupar el puesto de su mentora pero Marie-Louise Lachapelle eligió para su sucesión a otra alumna, una joven llamada Clémentine Hucherard ${ }^{18}$. Parece que Cadeau no quiso conformarse con ser una simple matrona y buscó otras opciones. Su oportunidad vino del Nuevo Mundo donde los Estados nacientes atraían a muchos europeos. De modo tal que Benita Paulina Cadeau y su esposo, Jean-Baptiste Fessel se embarcaron con dirección a la Nueva Orleans en 1823. La elección de esta ciudad estuvo motivada por su proximidad cultural y por sus vínculos personales, pues Benita Paulina Fessel llevaba consigo cartas de recomendación del profesor de la Facultad de Medicina de París, Antoine Dubois, dirigidas a Alexandre Labranche y a su hijo Octavio, presidente de la Cámara

\footnotetext{
15. Fessel, n. 13, p. III.

16. Beauvalet-Boutouyrie, n. 12, p.127.

17. Sus «Consejos a las mujeres encinta» se cierra así con un sentido homenaje a Marie-Louise Lachapelle, «mujer cuya memoria es bendecida por infinitas madres a quienes socorrió en el lecho del dolor y reverenciada por todas las matronas que tuvieron ventaja de recibir sus lecciones. Al acordarme de tan digna mujer, que guió particularmente mis estudios en el arte obstétrico, modelo de beneficencia y cuya modestia solo era igualable por su ciencia, no puedo contener lágrimas de pesar y gratitud», p. 37 .

18. Beauvalet-Boutouyrie, n. 12, p. 129.
} 
de representantes de Luisiana. También tenía otra carta dirigida al médico secretario de la Sociedad de Medicina de Nueva Orleans ${ }^{19}$.

No hay duda que Benita Paulina estuvo a la cabeza de esta empresa. De Jean-Baptiste Fessel no existen muchas referencias antes de llegar al Perú, sólo se sabe que era un oficial de sanidad, es decir que no era bachiller porque no había concluido sus estudios de medicina. El título de oficial de sanidad no le permitía ejercer fuera de su lugar de origen, es decir la ciudad de Lyon $^{20}$. De ahí probablemente que aceptara viajar con su esposa a América. Ésta última llevó las riendas del proyecto: las cartas de recomendación solo la nombraban a ella y no a su esposo. En un primer momento, la idea fue fundar una maternidad en Nueva Orleans, bajo el modelo de la de París. Pero este proyecto fracasó. Los Fessel se dirigieron entonces hacia el Sur y se instalaron en la ciudad mexicana de Guadalajara. En septiembre de 1824, Benita Paulina Fessel presentó ante el Cabildo una solicitud de creación de una Escuela de Partos ${ }^{21}$. La petición fue examinada a la vez que el Protomedicato se encargaba de comprobar la capacidad de la matrona. El resultado fue satisfactorio pues el cuerpo médico reconoció los excelentes conocimientos de Benita Paulina. A pesar de ello, el Cabildo de Guadalajara no consideró la creación de una Escuela de Partos como una prioridad de su gobierno. Rechazó la solicitud de la partera alegando tener importantes dificultades financieras. Por su parte, Benita Paulina no se quedó inactiva durante su estancia en México, más bien empleó su tiempo redactando una pequeña obra en español titulada Consejos a las mujeres encintas. Así, a pesar del entusiasmo y de la energía desplegada por la francesa para defender su causa, los Fessel tuvieron que resignarse a despedirse de México.

No se sabe por qué los esposos eligieron el Perú como nuevo destino. De cualquier modo, éstos ya se encontraban en Lima en 1826. Inmediatamente después de su llegada entraron en contacto con el Tribunal del Protomedicato ante el cual Madame Fessel expuso nuevamente su solicitud y presentó sus títulos y referencias académicas. El secretario general de esta institución era, en ese entonces, el doctor en medicina Miguel Tafur (1766-1833), rector de la Universidad de Lima, amigo personal y colabora-

19. Fessel, n. 13, p. III-V.

20. René, Louis. Médecine (Structure et organisation de la). In: Ambrière, Madeleine, dir. Dictionnaire du XIXe siècle européen. Paris: PUF; 1997, p. 739.

21. Díaz Robles; Oropeza Sandoval, n. 2, p. 242. 
dor del ministro del gobierno Hipólito Unanue. Ambos personajes estaban convencidos de la necesidad que tenía el nuevo Estado de formar matronas tituladas que se encargasen de remplazar a las tradicionales, esperando así asistir mejor a la población y aumentar el número de nacimientos. Gracias a su apoyo, el dossier de Madame Fessel fue aceptado por el Cabildo de Lima. Miguel Tafur e Hipólito Unanue quedaron tan convencidos de la pertinencia del proyecto que el ministro promulgó inmediatamente el decreto de creación de la Maternidad de Lima ${ }^{22}$. Los Fessel se instalaron en el local de un antiguo hospital militar mientras esperaban que el gobierno peruano inaugurase oficialmente la Maternidad de Lima ${ }^{23}$. Madame Fessel asumió el cargo de directora y recibió 1000 pesos para habilitar y equipar el nuevo local. El gobierno asignó a la Maternidad un presupuesto anual de 3.600 pesos, divididos en tres partes iguales ${ }^{24}$. El primer tercio iría al mantenimiento del hospital, el segundo a becas para las estudiantes, mientras que el tercer tercio correspondía al salario anual de la directora. Finalmente, para compensar la modesta asignación de la Maternidad, el gobierno ofreció a los Fessel un premio de 4.000 pesos suplementarios. El matrimonio tuvo sin embargo que esperar casi tres años para ver la instalación definitiva de la Casa de Maternidad de Lima. En mayo de 1830, los Fessel dejaron su clínica privada para instalarse en un edificio contiguo al Hospital de Mujeres (Hospital de la Caridad).

En el matrimonio Fessel, Benita Paulina fue siempre la más activa y emprendedora. Era una mujer instruida y con gran habilidad para escribir. Dejó una serie de documentos que fueron enviados a la prensa o a la administración aunque, como admitía ella misma, no dominaba perfectamente el español. Así, después de haber obtenido la autorización para ejercer emitida por el Protomedicato, la matrona publicó un folleto anunciando la apertura de su curso de obstetricia ${ }^{25}$. En varias oportunidades, envió artículos a los principales diarios de Lima para presentar el ejercicio de su arte y defender su profesión. Además de su actividad laboral, Madame Fessel consagró una parte de su tiempo libre a la redacción de libros de obstetricia: en los diez

22. Decreto del 10 de octubre de 1826, Archivo digital de la legislación en el Perú (ADLP). Disponible en: http:// www.congreso.gob.pe [citada 15 Abr 2011].

23. Rabí Chara, n. 3, p. 38.

24. Rabí Chara, n. 3, p. 45

25. Fessel, Benita Paulina. A las señoritas de la ciudad de Lima..., Lima; 1826. Folleto localizado en la Bibliothèque Nationale de France. 
años que pasó en el Perú, publicó cinco ${ }^{26}$. Este hecho es original y significativo pues existen pocos escritos de matronas en el mundo hispánico ${ }^{27}$. Dos de estas obras merecen especial atención. El Curso de partos se asemejaba a la mayoría de los libros de la época que tenían un carácter docente y vulgarizador. Se presentaba bajo forma de cartilla con preguntas y respuestas breves, de manera que las alumnas pudieran aprenderla de memoria. Este género literario se había difundido en toda Europa desde finales del siglo XVIII ${ }^{28}$. El curso de Madame Fessel se divide en tres partes: la primera trata de anatomía, la segunda de la teoría del parto natural y la tercera del «manejo de los partos explicado con el maniquí o demostración práctica de todas las posiciones en que puede hallarse el infante en el seno materno». La segunda obra, Práctica de partos, completa el volumen anterior pues presenta casos clínicos. Estas obras se publicaron sin necesidad de apelar a la autorización de su esposo como era frecuente en España en la misma época. En ningún momento Madame Fessel se mostró dependiente de la tutela de su esposo, fue más bien ella quien abogó para que Jean-Baptiste Fessel se convirtiera en el médico de la Maternidad ${ }^{29}$.

Infatigable y apasionada por su trabajo, Benita Paulina inauguró así la primera Maternidad de América Latina. La originalidad de esta institución era su asociación al Colegio de Partos, institución donde se ofreció una enseñanza de calidad.

26. Estas obras son: un Curso elemental de partos. Lima: Imprenta de la Liberdad (sic) por J. Masías; 1827, dos libros de casos clínicos titulados Práctica de Partos. Lima: Imprenta de J. Masías; 1830 y Relación histórica de una preñez extrauterina. Lima: Imprenta de J. Masías; 1830, así como una Relación del estado actual del arte obstetriz. Lima: Imprenta de José Masías; 1836. Por último, en 1827, Madame Fessel publicó una Reflexión sobre la organización de la Maternidad o Escuela de Partos en Lima (reproducida en Rabí Chara, n. 3) en la que defendía la importancia de su labor que había sido criticada por miembros del Cabildo de Lima. Las dos primeras obras están accesibles online en Digital Collections NLM: http://collections. nlm.nih.gov [citada 26 Mar 2012].

27. Como lo señala la historiadora Teresa Ortiz Gómez, en España solo se publicó una obra de ese tipo antes del siglo XX, el de Francisca Iracheta. Al respecto, veáse Ortiz Gómez, n. 1, 1999.

28. Así por ejemplo llegó a América la Cartilla Nueva, útil y necesaria para instruirse las matronas que vulgarmente se llaman comadres en el oficio de partear, escrita por el médico Antonio Medina por encargo del Protomedicato de Madrid en 1785 (reproducida por Kessinger Legacy Reprints).

29. Madame Fessel, en calidad de directora de la Maternidad, le pidió al embajador de Francia en Perú que intercediera ante el presidente peruano. Carta localizada en: Archivo General de la Nación (en adelante AGN), Lima, Ministerio de Ministerio de Justicia, Beneficencia e Instrucción 1826-1909 (R. J.), Beneficencia, Sección 4.7, serie Dirección General (1825-1857), L17. 


\section{Una formación teórica y práctica innovadora en el mundo hispánico}

Madame Fessel se inspiró en el modelo parisino, que conocía perfectamente, para organizar la Maternidad peruana. En un primer momento, las autoridades peruanas pensaron emplear a la matrona para dar clases de obstetricia a las parteras tradicionales. El director de la Beneficencia de Lima, Matías Maestro, le encargó al ministro del gobierno que conminara a las comadronas a presentarse a la Maternidad ${ }^{30}$. Sin embargo, ninguna de ellas se matriculó en los cursos sobre todo porque Madame Fessel tenía muy claro su proyecto. Para ella no se trataba de dar una clase general y propedéutica - para mujeres que, por lo general, no sabían ni leer ni escribir- sino de formar parteras altamente cualificadas que dispusiesen previamente de conocimientos básicos. Por eso es que desde el principio, las alumnas fueron más bien jóvenes con estudios primarios. La Maternidad de Lima albergó así a estudiantes, becadas o no, que siguieron clases en el Colegio de Partos adjunto al hospital. La primera promoción de parteras tituladas prestó juramento ante el Protomedicato en $1833^{31}$. De inmediato estas mujeres se situaron en una posición intermedia entre las comadronas y los médicos.

Desde un principio, Benita Paulina Fessel percibió el interés de situarse bajo la égida de la representación ilustrada de la maternidad. Por eso adoptó e hizo suyo el discurso de los ilustrados peruanos. En sus obras, la matrona francesa se muestra tan intransigente con las comadronas como lo habían sido los colaboradores del Mercurio Peruano y como lo eran muchas parteras europeas de la misma época. En ningún momento reivindicó algún vínculo con las parteras tradicionales, por el contrario se distanció completamente de ellas:

«Me abstengo de nombrarlas, y las compadezco, porque destituidas de toda instrucción, son absolutamente incapaces de abreviar los dolores y la duración del parto, y principalmente de saber cómo y cuándo deberá nacer la inocente criatura expuesta a perecer en sus manos, porque no recibe el pequeño auxilio que necesita y reclama en vano algunas veces» 32 .

30. Maestro, Matías. Carta a: Ministro de Gobierno y Relaciones Exteriores. 26 Sep 1827. 1 hoja. AGN, Ministerio de Justicia, Beneficencia e Instrucción 1826-1909 (R.J.), Sección 4.7, serie Dirección General (1825-1857), L17.

31. Rabí Chara, n. 3, p. 49.

32. Fessel, n. 26; 1830, p. V. 
Así, en sus escritos, Madame Fessel condenó abiertamente el carácter empírico y supersticioso de la práctica de las llamadas recibidoras. Pero no por ello se remitió totalmente a los médicos. Para Madame Fessel, en materia de partos, las más habilitadas eran las parteras tituladas como ella. Además, se percató rápidamente de que los médicos peruanos estaban menos capacitados que ella en lo que a la obstetricia se refería, principalmente por falta de experiencia clínica, como señalaba ella misma:

«Sin tratar de despreciar ni disminuir el mérito de ninguna de las personas que ejercen la profesión médica en Lima, sería fácil probar que esta ciudad no está provista de comadrones dotados de los conocimientos positivos que exige esta última profesión, a menos que se quiera suponer que la incertidumbre, la temeridad o algunas veces el acaso, puedan suplir los sabios e indispensables preceptos de la teoría aplicada a la sana práctica» ${ }^{33}$.

Pero la matrona francesa va más allá. Para ella los médicos, a pesar de los altos conocimientos científicos que poseían, no debían encargarse del arte de partear. Sus Consejos a las mujeres encintas se abren con una cita de la obra del médico francés Philippe Hecquet, escrita en 1707 y cuyo título resume perfectamente la posición de Madame Fessel: De l'indécence aux hommes d'accoucher les femmes. Al concluir su obra, la matrona vuelve a desarrollar su idea esta vez basándose en el famoso libro de otro médico francés, Pierre Roussel, llamado Le système physique et moral de la femme, y publicado a principios del siglo XIX. Madame Fessel hizo suyas las frases del libro que aludían al tema de los partos:

«Aunque la operación de partear pertenece al arte de curar, sin embargo su ejercicio no es propio del hombre. El carácter de decencia que exige esta función, la confianza absoluta que naturalmente deben tener entre sí los individuos de un mismo sexo, en fin, todo llama a las mujeres a ejercerla, y parece serlas este empleo más propio, cuanto que se hallan dotadas de todas las ventajas necesarias para llenarlo con suceso. Se sabe con cuanta maña y destreza sus manos pequeñas y suaves se resbalan, se insinúan por todas partes sin inconvenientes, penetrando hasta la fuente del mal sin aumentarlo y llevando a la parte enferma un remedio cierto sin renovar dolores ya adormecidos» ${ }^{34}$.

33. Fessel, n. 26; 1830, p. V.

34. Traducción de Benita Paulina Fessel de la obra de Roussel, Pierre. Le système physique et moral de la femme. Paris: Crapart, Caille et Ravier; 1803, p. 217. 
Madame Fessel consideraba que el parto debía quedarse en la esfera femenina, y otorgó a las matronas un lugar preponderante. Toda su obra prueba esa voluntad de erigir la profesión de matrona al nivel de un arte, es decir un oficio que exige una aptitud, una técnica y conocimientos racionales que en este caso sólo las mujeres pueden dominar. De ahí que se inscribiera en el linaje de las parteras que contribuyeron ampliamente a los progresos de la ciencia obstétrica, tales como Louise Bourgeois (15631636), matrona de María de Medicis, Marguerite du Tertre de la Marche (1638-1706), matrona jefa del Hôtel-Dieu de París, Angélique Marguerite le Boursier du Coudray (1712-1789), primera partera en utilizar los maniquís en sus cursos de obstetricia, Marie-Jonet Dugés (1730-1797), Marie-Louise Lachapelle (1769-1821) y Marie Anne Victorine Boivin (1773-1847), estas tres últimas matronas jefas en los hospitales parisinos ${ }^{35}$. Esta identificación con un linaje de parteras tiene como objetivo realzar la importancia de la profesión. Todas estas mujeres figuraban como especialistas del arte obstetriz. Madame Fessel emplea constantemente este adjetivo que transforma en sustantivo y que se ha perpetuado en el vocabulario actual peruano. Tanto ella como sus discípulas no se autodenominaron ni matronas ni parteras, sino que prefirieron emplear ese neologismo para designarse. El término obstetriz, derivado directamente del latín obstetrix, le permitía romper con la profesión de las parteras tradicionales y crear una nueva que definió en estos términos:

«Confesemos que este arte no se puede aprender sino en su escuela. La demostración práctica que exige, según la diversa posición del feto; el uso de los instrumentos en los partos contra naturales y laboriosos para salvar la madre y el hijo es indispensable, y su manejo no se puede adquirir sin el maniquí y el estudio de las piezas anatómicas, preparadas y colocadas al intento ${ }^{36}$.

35. Varios estudios han sido dedicados a las parteras francesas. En lo que respecta a Louise Bourgeois, veáse: Sheridan, Bridgette. At birth: the modern state, modern medicine, and the royal midwife Louise Bourgeois in seventeeth-century France. Dynamis. 1999; 19, p. 145-166; Vons, Jacqueline, La parole d'une sage-femme: Louise Bourgeois (1563-1636). In: BoudonMillot, Véronique, Dasen, Véronique, Maire, Brigitte, eds. Femmes en médecine en I'honneur de Danielle Gourevitch. Paris: De Boccard Edition-Diffusion; 2008, p. 223-238. Sobre Madame du Coudray veáse: Rattner Gelbart, Nina. The king's midwife. A history and mystery of Madame du Coudray. Berkeley-Los Angeles-Londres: University of California Press; 1998; Midwife to a nation: Mme du Coudray serves France. In: Marland, n. 1, 1993. Sobre las matronas francesas del siglo XIX, veáse Beauvalet-Boutouyrie, n. 12, p. 127-134.

36. Fessel, n. 26; 1827, p. 10. 
Como se ve en este fragmento, el conocimiento de la anatomía fue el núcleo de la enseñanza de la Escuela de Partos de Lima. Disponer de ese saber propiamente médico y racional permitió a las obstetrices peruanas distinguirse de las matronas tradicionales. De hecho, el programa de estudios de las alumnas obstetrices no solo correspondía al curso de obstetricia de los estudiantes de la Facultad de Medicina de Lima. Se trataba en realidad de un curso teórico más completo, pues las futuras parteras estudiaban durante cuatro años lo que los futuros médicos hacían en uno. Los médicos de la época replicaban que la duración de los estudios de las mujeres se debía a su ineptitud y a la necesidad de avanzar más despacio que los varones, hábiles y rápidos por esencia. Finalmente, sin embargo, las alumnas de la Escuela de Partos recibían una importante formación teórica que correspondía principalmente al estudio de la anatomía. Las futuras obstetrices veían así los mismos temas obstétricos que los estudiantes varones, a saber: los partos naturales y distócicos y las enfermedades de las parturientas y de los recién nacidos. Madame Fessel exigía además a sus alumnas que aprendieran francés para poder leer las obras de los grandes obstetras, que generalmente estaban escritas en esa lengua. Esta obligación quedó en vigencia aún después de que Madame Fessel retornara a Francia ${ }^{37}$. Las alumnas tenían además acceso a una serie de libros traídos especialmente de Europa. De hecho, en la biblioteca de la Escuela de Partos de Lima figuraban los libros de Claude-Martin Gardien ${ }^{38}$, Jean-Louis Baudelocque ${ }^{39}$ y Joseph Capuron ${ }^{40}$. Se agregaban a estas obras las de los obstetras más célebres del siglo XVIII: André Levret ${ }^{41}$, William Smellie ${ }^{42}$ y Johann Georg

37. Los reglamentos de la Maternidad de Lima de 1865 y de 1872, reservan una plaza a una profesora de francés. Dichos documentos están disponibles en la Biblioteca del Instituto Riva-Agüero (Lima).

38. Gardien, Claude-Martin. Traité des Accouchements, des maladies des femmes, de l'éducation médicinale et des maladies propres à cet âge. Paris: Crochard; 1807, 4 vols.

39. Baudelocque, Jean-Louis. Principes sur l'art des accouchements, par demandes et réponses, en faveur des élèves sages-femmes, 4a ed. Paris; 1812.

40. De este autor figuran dos obras: Capuron, Joseph. Cours théorique et pratique des accouchements. Paris: Chez l'auteur; 1811 y Traité des maladies des femmes, depuis la puberté jusqu'à I'âge critique inclusivement, Paris; Chez l'auteur [\&] Croullebois, 1812.

41. Levret, André. L'Art des accouchemens, démontré par des principes de physique et de méchanique, pour servir d'introduction et de base à des leçons particulières, $3^{a}$ ed. Paris: P.-Fr. Didot le jeune; 1766; Observations sur les causes et les accidens de plusieurs accouchemens laborieux. Suite des Observations... Paris: T. Barrois; 1780.

42. Smellie, William. Observations sur les accouchements, ou Suite de la théorie et pratique de cet art. Paris: P. F. Didot le jeune; 1777. 
Roederer ${ }^{43}$. A estas obras se agregaban los manuales redactados por las matronas francesas Marie-Louise Lachapelle ${ }^{44}$ y Madame Fessel ${ }^{45}$.

El método de enseñanza asociaba aspectos clásicos a otros más innovadores. En primer lugar, el aprendizaje se centraba en la memorización y la repetición: las alumnas obstetrices tenían así que aprender de memoria respuestas a las preguntas presentadas en clase. El objetivo de este método era crear automatismos a la manera de la pedagogía escolástica. Como se acostumbraba en la Maternidad de Port-Royal, existía en Lima un sistema enseñanza mutua y de repaso de las clases. Las alumnas mayores hacían de repetidoras para las más novatas ${ }^{46}$. Además, Madame Fessel asoció al tedioso y abstracto trabajo de memorización y repetición una serie de ejercicios prácticos. La matrona empleó en sus clases maniquís y láminas que había hecho traer a su escuela especialmente desde Francia. También presentaba a sus estudiantes fetos conservados en vino ${ }^{47}$. Hay que resaltar el carácter singular de esta enseñanza en el Perú sobre todo para jóvenes que, en algunos casos, apenas habían seguido clases con preceptores encargados de enseñarles la gramática, la historia, la geografía o los buenos modales pero casi nunca clases de ciencias naturales. Además, las clases se llevaban a cabo en el anfiteatro de la Escuela de Medicina. La profesora de partos se encargaba de la parte descriptiva mientras que la «disección de órganos, inyecciones de los vasos, maceraciones de las partes blandas o huesosas ${ }^{48}$ correspondía al médico jefe de la Maternidad. Cabe señalar que esta formación teórica fue un factor de distinción social. Pero al mismo tiempo esta diferenciación y valoración provocó una actitud de suspicacia y de envidia por parte de ciertos miembros del cuerpo médico que trataron

43. Roederer, Johann Georg. De Axi Pelvis Programma quo... Ad orationem benevole audientiam invitat, et simul lections suas denuo indicit. Göttingen: Schulzius; 1751.

44. Lachapelle, Marie-Louise. Pratique des accouchemens ou Mémoires et observations choisies sur les points les plus importans de l'art, Paris: J.-B. Baillière; 1821.

45. Esta lista figura en el expediente de Mme Benita Paulina Cadeau de Fessel seguido en 1836 sobre la venta de sus bienes y libros, antes de marcharse a Francia. Reproducido en: Rabí Chara, n. 3, p. 328.

46. Beauvalet-Boutouyrie, n. 12, p. 163.

47. Gracias al inventario realizado cuando Madame Fessel partió del Perú sabemos que la escuela disponía de "cinco fetos en espíritu de vino», así como láminas y maniquíes destinados a la enseñanza. Rabí Chara, n. 3.

48. Fessel, n. 26; 1827; p. 13. 
de denigrar a las parteras criticando su falta de cultura y de instrucción ${ }^{49}$. Esta animosidad se explica por el hecho que las obstetrices poseían una experiencia clínica de la que carecían en aquella época la mayoría de los médicos, aun los más reconocidos. Durante los cuatro años que duraba la formación, las alumnas parteras asistían entre unos cuarenta a cien partos mientras que los estudiantes de último año de medicina debían contentarse con presenciar como máximo una decena de casos.

Así, en el Perú, la profesión de obstetriz se estableció y se organizó gracias al dinamismo y a la perseverancia de Benita Paulina Fessel, así como al gobierno peruano que percibió la oportunidad de efectuar un control sobre la maternidad. Pero la matrona regresó a Francia en 1836, agobiada probablemente tanto por no tener el reconocimiento que esperaba como por problemas de salud. El paso de Madame Fessel por el Perú abrió las puertas a un número cada vez más importante de mujeres deseosas de tener una profesión reconocida socialmente.

\section{Desarrollo de la profesión de obstetriz bajo la tutela de los médicos}

El siglo XIX se presenta como la edad de oro de la profesión de obstetriz en el Perú. Siguiendo los pasos de Madame Fessel, un número creciente de mujeres optó por los estudios de obstetricia (ver figura núm. 1). Así, mientras que en 1833 solo había cinco obstetrices formadas por Madame Fessel, en 1902 figuraban 211 como matronas tituladas y habilitadas a ejercer en toda la República del Perú ${ }^{50}$.

\footnotetext{
49. Tenemos referencia de quejas de algunos profesores a través de la prensa médica, como por ejemplo: Crónica Médica. Lima: Imprenta y Librería de San Pedro; 1900, p. 296.

50. Anales Universitarios del Perú. Tomo XXX. Lima: Imprenta Liberal; 1903.
} 
Figura 1. Alumnas de la Escuela de Partos de Lima (1833-1902)

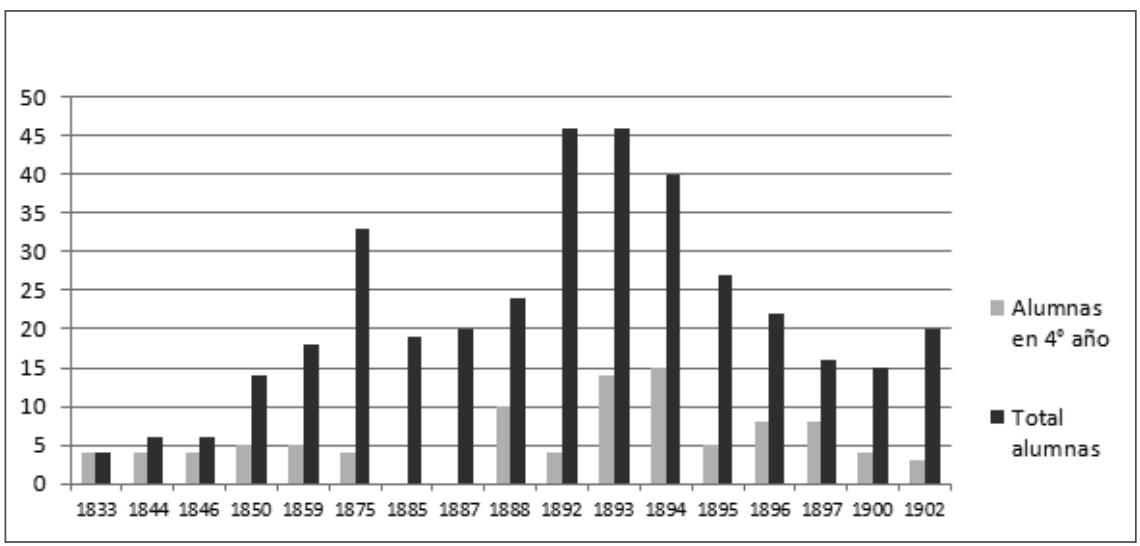

Fuente: Elaboración propia ${ }^{51}$.

La formación impartida por la escuela siguió siendo de las más rigurosas, con la clásica asociación de la teoría y de la práctica, hasta el punto que la Escuela de la Maternidad de Lima se alzó al nivel de modelo y se exportó a otros países de la región. Mientras que en México los estudios de obstetricia duraban dos años, en el Perú seguían siendo de cuatro de manera que las alumnas disponían del tiempo necesario para asimilar y profundizar los conocimientos obstétricos además de asistir a un número importante de partos. Con la promulgación del Reglamento de Instrucción, el plan de estudios del colegio se asienta de manera definitiva. Según éste, las alumnas seguían cursos de anatomía en primer año, de fisiología de partos en segundo año, de teoría del parto natural en tercer año y de teoría de los partos distócicos o artificiales en cuarto año. Acompañaban estos cursos la clínica obstétrica, la sangría y la vacuna, que se repetían durante los cuatro años de estudios ${ }^{52}$.

La partera jefa, las profesoras de obstetricia así como las alumnas internas residían en la Maternidad y por lo tanto acompañaban muy de cerca a las

51. Cuadro elaborado a partir de diversas fuentes: Fessel, n. 26; Gaceta Médica de Lima, años 1857-1867. Localizada en: Biblioteca de la Universidad Mayor de San Marcos (Lima); Gaceta Médica. Lima: Imprenta del Estado, años 1875-1879; Crónica Médica. Lima: Imprenta del Universo de Carlos Príncipe, años 1884-1901; Anales Universitarios del Perú, Lima: Imprenta Liberal; tomos XIV (1888), XVI (1890), XX (1896), XXII (1897), XXX (1903).

52. Reglamentos de la Facultad de Medicina de 1856 y 1886, disponibles en: ADPL, n. 22. 
parturientas. Hasta los años 1880, los estudiantes - varones-de medicina no recibían durante sus estudios que duraban siete y luego seis años, más que un curso de obstetricia titulado «partos y enfermedades puerperales» en general en el último año. La clínica, en cambio, siguió siendo bastante limitada pues la moral de la época - rigurosamente impuesta por las religiosas encargadas de la administración de los hospitales de mujeres- y el pudor de las parturientas restringieron la presencia de los varones en las salas de parto. Durante casi todo el siglo XIX, las mujeres poseyeron una mayor competencia en materia obstétrica; aun cuando se las mantuvo bajo la vigilancia de los médicos, debieron ser conscientes de su papel social y de su poder.

Contrariamente a las matronas tradicionales, las obstetrices del siglo XIX no pertenecieron a categorías sociales bajas. En muchos casos se trataba de mujeres de la pequeña burguesía criolla o mestiza. Antes de empezar su formación profesional, habían terminado sus estudios primarios y tenían por lo general un buen nivel de preparación. Eran también bastante jóvenes - muchas tenían entre 15 y 18 años en el momento de ingresar- y no había obligación que estuvieran casadas como en el caso español ${ }^{53}$. Así por ejemplo, Irene Salinas, que se presentó en 1882, tenía 17 años de edad y disponía de un certificado de su profesor demostrando que había cursado toda su primaria y estudiado religión, gramática española, aritmética y geografía. Además, había seguido clases de economía doméstica, de historia del Perú y de historia natural y completó también su formación con clases de francés $^{54}$. En ese sentido, en Lima los estudios de obstetricia fueron selectivos por lo que marginaron a las matronas tradicionales, en general iletradas y poco instruidas, y sin capacidad para pasar el examen de admisión. Las candidatas se presentaban así ante un profesor de la Universidad encargado de evaluarlas en las materias anteriormente referidas ${ }^{55}$. Una vez admitidas en la Escuela, las alumnas pasaban cada año un examen que descartaba, según las promociones, entre el 10\% y el 15\% de ellas.

A finales del siglo XIX, la selección se acentuó y las autoridades académicas solicitaron diplomas de estudios oficiales. De ahí que algunas candidatas fueran hasta maestras de escuela. Algunas de ellas parecían haberse

53. Ortiz Gómez, n. 1; 1999, p. 63.

54. Expedientes de matrículas. Caja 1571. Archivo Histórico Domingo Angulo, Facultad de Medicina, Lima.

55. Expedientes de matrículas, n. 54. 
preparado muy bien, estudiando francés u otras materias complementarias para alcanzar la admisión. La escuela aceptaba también a obstetrices extranjeras como en el caso de Clara Coste de Viellefon, profesora de partos de París y de Santiago de Chile, que solicitó su incorporación en $1855^{56}$.

Todo esto tiende a probar que la profesión de obstetriz en el Perú del siglo XIX iba asociada a una buena valoración social. Hasta finales de siglo fue la única posibilidad para las mujeres de tener acceso a un diploma universitario reconocido a nivel nacional. $Y$ aunque los estudios de obstetricia no se encontrasen al mismo nivel que los de medicina, la universidad se convirtió en un espacio de descubrimiento e interacción entre géneros. Para las futuras obstetrices, el paso por la universidad constituyó una verdadera apertura intelectual y humana. En 1895, la Escuela de Partos se integró definitivamente a la Facultad de Medicina. Las alumnas se convirtieron entonces en verdaderas universitarias, frecuentando los mismos locales de la Facultad y asistiendo a algunas clases con los varones. Se trató de un primer paso importante para las jóvenes, que empezaron a ver la posibilidad de seguir estudios en las facultades tradicionales ${ }^{57}$.

Por otro lado, la profesión de partera titulada gozó del apoyo del naciente Estado peruano. Los diferentes gobiernos, acosados por el temor a la despoblación, emplearon a las obstetrices como difusoras de las teorías higienistas en pleno auge, a la vez que se apoyaron en ellas para controlar de alguna manera la maternidad y por ende a las mujeres. Así, gracias al establecimiento de becas, las alumnas de provincias (una por departamento) podían seguir los estudios de obstetricia en Lima, esperando seguramente que al finalizarlos regresarían a ejercer su profesión en su lugar de origen. Del mismo modo, una vez diplomadas, varias obstetrices fueron enviadas a provincias para ejercer su profesión y formar a otras parteras. Tal fue el caso de Jacoba Gómez que se recibió en 1855 y años después, en 1866, fue nombrada por el gobierno profesora de partos del hospital de Huánuco con la obligación de asistir a las indigentes y de formar a cuatro jóvenes designadas por la Sociedad de Beneficencia. El gobierno se comprometió

\footnotetext{
56. Expedientes de matrículas, n. 54.

57. Laura Esther Rodríguez Dulanto, fue así la primera mujer peruana en ingresar a la Facultad de Ciencias en 1892 y graduarse de médico en 1900.
} 
a pagarle un sueldo mensual y hasta se encargó de los gastos de transporte e instalación ${ }^{58}$.

La Escuela de Partos se convirtió de esta manera en el primer establecimiento obstétrico del Perú. Durante la segunda mitad del siglo XIX, recibió en sus aulas un promedio de veinte alumnas por año, con picos que alcanzaron hasta unas cuarenta después de la Guerra del Pacífico, entre 1892 y 1894. Las alumnas eran principalmente originarias del departamento de Lima, con extensiones hasta Ica y algunas otras regiones de la costa del Perú. La Escuela formó un promedio de seis obstetrices por promoción. Y a partir de 1863, otras Facultades de Medicina se fueron creando en el Perú. Las primeras se instalaron en el Cusco y Arequipa, lo que supuso la apertura de cursos de obstetricia en estas dos ciudades. Para dictar las clases de obstetricia en estos nuevos centros de estudios, la Sociedad de Medicina eligió a dos antiguas alumnas de la Escuela de Partos, ambas de 26 años de edad y oriundas de la costa norte del Perú. Emilia Rambla fue nombrada para enseñar en el Cusco, mientras Micaela Cabrera se instaló en la Escuela de Partos de la Universidad de Arequipa ${ }^{59}$. A esta proyección nacional, hay que añadirle otra, esta vez internacional. En efecto, dos discípulas de Madame Fessel, Juana Reyes y Cipriana Dueñas salieron del Perú para establecer escuelas de obstetricia, siguiendo el modelo limeño, en La Paz y en Quito respectivamente ${ }^{60}$.

De esta manera, la segunda mitad del siglo XIX fue testigo de una verdadera multiplicación de las parteras tituladas que trataron de afianzarse como las especialistas de la obstetricia, fieles a la enseñanza de Madame Fessel. De forma cada vez más afirmada, las mujeres pudientes y de clase media las solicitaban prioritariamente durante el embarazo y en el momento del parto. Sin embargo, los médicos veían con malos ojos el poder que habían alcanzado las obstetrices diplomadas en la Maternidad de Lima. Como reacción, éstos tendieron a denigrar el trabajo de las parteras así como su capacidad, al tiempo que se inmiscuían cada vez más en todo lo respecta la ginecología y la obstetricia. Las publicaciones médicas peruanas prueban el interés creciente por estos temas así como una gran avidez hacia

58. AGN, Ministerio de Justicia, Beneficencia e Instrucción (R. J.), Sección: 3.8, Serie: Dirección General de Estudios, 1855-1865.

59. Expedientes de título (Matrona, Obstetriz). Caja 4. Archivo Domingo Angulo, Facultad de Medicina, Lima.

60. Rabí Chara, n. 3, p. 325 
las novedades científicas venidas del extranjero, principalmente de Francia. Muchos de los médicos viajaron a París para capacitarse, enviados a veces por el gobierno y otras por cuenta propia. Seguros de su capacidad y de su saber científico, no dudaron en imponer su visión del parto sin tener en cuenta la peculiaridad de sus pacientes, como lo muestra el siguiente fragmento de una tesis de 1908 sobre la Maternidad de Lima:

«Muchas se resisten a dar a luz en la posición dorsal, prefiriendo la actitud de rodillas y no en el lecho sino en el suelo, adonde colocan cualquier lienzo; cuando se les sorprende en esta posición y se les obliga al decúbito manifiestan su desagrado para acostarse diciendo que así el cuerpo no las ayuda. Cuando concurríamos a la clínica tuvimos ocasión de ver entre otros casos, el de una muchacha indígena de 16 años, que vino al servicio ser asistida de su primer parto; conducida al lecho de reconocimiento se resiste tenazmente a ser examinada por nosotros; después de haber agotado consejos persuasivos y hasta amenazas para lograr nuestro objeto, termina por abandonar el servicio, sino era asistida por una mujer. Su estado de indigencia la obliga a volver a los pocos días, cuando se iniciaran los dolores del parto y con tan poca fortuna para ella que ingresó durante las horas de la clínica» ${ }^{61}$.

En la Maternidad de Lima, como en otros hospitales peruanos donde ejercieron, las obstetrices se mantuvieron bajo la tutela de los médicos y tuvieron probablemente en muchos casos que someterse a su manera de practicar la medicina. Pero muchas parteras, una vez tituladas, dejaron los centros hospitalarios y ejercieron su profesión de manera liberal, en Lima y en provincias. Recibían a sus pacientes a veces en sus consultorios privados pero en la mayoría de los casos las atendían a domicilio.

\section{Conclusión}

En el Perú, la profesión de obstetriz surgió en un contexto de difusión de las ideas obstétricas e higienistas así como de creación del Estado peruano. Madame Fessel cumplió un papel determinante en la creación de una Maternidad y de la Escuela de Partos que le fue asociada siguiendo el modelo de la Maternidad de Port-Royal. En ella se formaron, durante todo

61. Moloche, Ricardo. La Maternidad de Lima. Contribución a la historia de la Obstetricia. Tesis de Bachillerato, Facultad de Medicina. Lima: Imprenta del Estado; 1908, p. 9. 
el siglo XIX, cientos de obstetrices, altamente cualificadas tanto en teoría como en práctica. La originalidad peruana, en relación a otros países hispánicos y europeos, reside en el lugar importante y relativamente autónomo que tuvieron las parteras tituladas. No necesitaron situarse bajo la tutela de un esposo para estudiar y ejercer. Si bien es cierto que la ofensiva de los médicos por controlar el momento del parto fue sensible durante todo el siglo XIX, las obstetrices peruanas no se doblegaron y su poder simbólico fue sólido hasta mediados del siglo XX, contrariamente a lo que sucedía en la misma época en otros países como España. En los hospitales, fueron ellas quienes acompañaron a las parturientas pues los médicos no residían en las maternidades. Todos los partos normales - es decir la gran mayoría- les eran delegados pues los facultativos se reservaban los distócicos. Además, hasta los años 1960, la mayoría de las peruanas daban a luz en casa, acompañadas de una obstetriz.

Las obstetrices participaron así, de manera activa, en la divulgación y transmisión de los saberes obstétricos. Aunque nunca reivindicaron una autoridad superior a la de los médicos, a los que siempre recurrieron en los casos difíciles, crearon poco a poco una autoridad propia, reconocida socialmente. Este proceso trajo consigo el desprestigio cada vez mayor de las parteras tradicionales. Durante el siglo XX, el número de obstetrices fue creciendo en las grandes ciudades mientras que disminuía el de las comadronas.

Los diferentes ejemplos a los que hemos aludido en este artículo muestran que existió una importante movilidad - a diversas escalas- de las parteras del siglo XIX. Las fuentes indican que al menos tres obstetrices francesas optaron por el Perú para ejercer su profesión. La Maternidad de Lima se convirtió rápidamente en modelo regional y un puñado de matronas salió del Perú con destino a otros países andinos. Pero el movimiento de mayor amplitud fue el que condujo a un número creciente de obstetrices formadas en Lima a provincias más o menos alejadas de la capital. Pero esta historia de la movilidad y de las redes obstétricas que ellas establecieron está todavía por hacer. 


\section{ARTICLES}

\section{From the traditional midwife to the female obstetrician. Birth and pinnacle of the profession of licensed midwifery in 19th century Peru}

Lissell Quiroz ................................................

1.-Introduction. 2.-Inheritance of the Enlightenment: denigration of the mid-wife and development of the obstetrician. 3.-Benita Paulina Cadeau, the Peruvian «Madame Lachapelle». 4.- Theoretical training and innovatory practice in the Hispanic world. 5.-Development of the profession of obstetrics under the supervision of physicians. 6.-Conclusion.

ABSTRACT: In Peru and the rest of the Hispanic world, the 19th century was a time of profound change in the practice of medicine and especially in obstetrics. Among the leading agents in this development were the midwives, whose profession was born and established at that time. Previously, traditional midwives were responsible for accompanying women during labour in an almost entirely female universe. In the late 18th century, enlightened Peruvians became interested in child-birth and the perinatal period. They mainly criticized the practice of traditional mid-wives and presented to public opinion the need for theoretical training under the supervision of medical staff. After Independence, the Enlightenment discourse was resumed by the public authorities of the nascent state. This project became a reality thanks to the arrival in Peru of the French midwife, Benita Paulina Fessel, an enterprising woman who wished to establish the Parisian (Port-Royal) birth model. The confluence of these factors led to the birth in 1826 of the first Maternity Hospital in the Hispanic world with a specific function, which was associated with a Childbirth school led by Madame Fessel, who was herself an alumna of the Maternity Hospital in Paris. Lima Maternity Hospital trained several generations of midwives, who received an excellent theoretical and practical training and established themselves as competent professionals. During the second half of the 19th century, obstetricians, as they were named from that time in Peru, accompanied more and more women in the throes of childbirth and proved able to endure the pressure of doctors and take possession of this essential moment in the life of women. 